\title{
Quality Signaling by Location in Market for Search Goods
}

\section{J. WU}

\author{
Department of Mathematics, Jianghan University, Wuhan, China
}

\begin{abstract}
This paper discusses the quality signaling in the location game considering search cost. We comment on Paul W.J. de Bijl's paper published in the International Journal of Industrial Organization and point out that the proof of equilibria in the original article was not complete. We also question the search cost being considered as a variable cost in the original article, and suggest that it is more reasonable to consider it as a fixed cost. The detailed proof is give in the paper and future research ideas are provided.
\end{abstract}

KEYWORD: Location game; signaling theory; search cost

\section{INTRODUCTION}

"Entry deterrence and signaling in markets for search goods" is a paper written by Paul W.J. de Bijl and published in the International Journal of Industrial Organization [1]. This paper focused on search goods market where consumers can observe the quality of goods before buying, so that a high-quality entrant may have an advantage on market entry. The author showed that if the incumbent does not know the entrant's type (Model I), when search cost is low, the entrant can signal high quality by choosing a sufficient high price; while when search cost is sufficiently high, there will be a entry barrier for the potential entrant. On the other hand, if the incumbent observes the entrant's type (Model II), the entrant can rely on its rival's price to inform consumers its quality, so that it has a large degree of freedom in choosing its price. Therefore the incumbent cannot exploit information about the entrant in a profitable way. The author showed the pooling equilibria and separating equilibria under two different models. At last the author pointed out a couple of potential extensions of this research. One would be to consider the choice of location as a quality signal. The other would be to allow the incumbent to spy on an entrant to observe its quality.

\section{DISCUSSION}

Meanwhile, we have several concerns about the paper itself. First, the way that the author proved the equilibria was awkward. He did not show when one player deviates from the equilibrium, the (expected) payoff of that player will not increase, which is the normal way we show equilibrium. Therefore we redo the proof of the separating equilibrium under model I as an example in the following way:

Proposition 1: Under Assumptions 1 and 2, for any $\delta$, there exists a unique separating equilibrium:

(i) if $\delta>\delta_{1}$, then a high-quality firm enters; $p_{1}^{*}=p_{2}^{*}\left(q_{L}\right)=c_{L}$ and $p_{2}^{*}\left(q_{H}\right)=c_{L}+q_{H}-q_{L}$; $\pi_{1}^{*}=0$ and $\pi_{2}^{*}=\alpha\left(c_{L}+q_{H}-q_{L}-c_{H}\right)$;

(ii) if $\delta \leq \delta_{1}$, then the incumbent deters entry of a high quality firm; $p_{1}^{*}=p_{2}^{*}\left(q_{L}\right)=c_{L} \quad$ and $p_{2}^{*}\left(q_{H}\right)=c_{L}+q_{H}-q_{L} ; \pi_{1}^{*}=\pi_{2}^{*}=0$;

where we assume $q_{H}-q_{L} \leq q_{L}-c_{L}$, define $\delta_{1} \equiv 1-\frac{q_{H}-q_{L}}{q_{L}-c_{L}}$, and

Assumption 1: Given an equilibrium price $p_{2}^{*}\left(q_{2}\right)$, the consumer's belief satisfies

$$
\mu\left(p_{1}, p_{2}^{*}\left(q_{2}\right)\right)=\mu\left(p_{1}{ }^{\prime}, p_{2}^{*}\left(q_{2}\right)\right) \quad \text { for }
$$
all $p_{1} \neq p_{1}^{\prime}$.

Assumption 2: Given an equilibrium price $p_{1}^{*}$, the consumer's belief satisfies $\mu\left(p_{1}^{*}, p_{2}\right)=1$ for all $p_{2}$ such that $q_{L}-p_{2}<\delta\left(q_{L}-p_{1}^{*}\right)$.

Proof: Under Assumption 1 and 2 , for $\mathcal{E}>0$, 
(i) if $\delta>\delta_{1}$, then

$$
\begin{aligned}
& \pi_{1}\left(c_{L}+\varepsilon, c_{L}, c_{L^{+}} \quad q_{\bar{H}} \quad q \overrightarrow{\bar{D}}\right. \\
& 0=\pi_{1}\left(\begin{array}{lllll}
c_{L} & \mathcal{C}_{L} & \mathcal{c}_{L}+ & q_{\bar{H}} & q_{L}
\end{array}\right) ; \\
& \pi_{1}\left(c_{L}-\varepsilon, c_{L}, c_{\bar{L}}+q_{\bar{H}} \quad q_{\bar{L}}\right. \\
& -\varepsilon<0=\pi_{1}\left(\begin{array}{llllll}
c_{L} & c_{L} & c_{L}^{+} & q_{\bar{H}} & q ;
\end{array}\right. \\
& \pi_{2}\left(c_{L}, c_{L}+\varepsilon, c_{L^{+}} \quad q_{\bar{H}} \quad q_{\vec{L}}\right. \\
& 0=\pi_{2}\left(\begin{array}{lllll}
c_{L} & \mathcal{C}_{L} & \mathcal{C}_{L}^{+} & q_{\bar{H}} & q_{L}
\end{array}\right) ; \\
& \pi_{2}\left(c_{L}, c_{L}-\varepsilon, c_{L^{+}} \quad q_{\bar{H}} \quad q_{\bar{D}}\right. \\
& -\varepsilon<0=\pi_{2}\left(\begin{array}{llllll}
c_{L} & c_{L} & c_{L}^{+} & q_{\bar{H}} & q \text {; }
\end{array}\right. \\
& \pi_{3}\left(c_{L}, c_{L}, c_{L}^{+} \quad q_{\bar{H}} \quad q_{L} \varepsilon\right)= \\
& 0<c_{L}+q_{H}-q_{\bar{L}} \quad c_{\bar{H}} \\
& \pi_{3}\left(c_{L}, c_{L}, c_{L^{+}} \quad q_{\bar{H}} \quad q_{L}\right) \quad ; \\
& \pi_{3}\left(c_{L}, c_{L}, c_{L}^{+} \quad q_{\bar{H}} \quad q_{L} \varepsilon\right)= \\
& 0<c_{L}+q_{H}-q_{\bar{L}} \quad c_{\bar{H}} \\
& \pi_{3}\left(c_{L}, c_{L}, c_{L}^{+} \quad q_{\bar{H}} \quad q_{L}\right) \quad ;
\end{aligned}
$$

where the last inequality is true because under Assumption 2, we have

$$
\begin{aligned}
& p_{2}\left(q_{H}\right)>q_{L}-\delta\left(q_{L}-c_{L}\right)>q_{L}-\delta_{1}\left(q_{L}-c_{L}\right)= \\
& q_{L}-\left(q_{L}-c_{L}-q_{H}+q_{L}\right)=c_{L}+q_{H}-q_{L}
\end{aligned}
$$

then if $p_{2}\left(q_{H}\right)=c_{L}+q_{H}-q_{L}-\varepsilon<c_{L}+q_{H}-q_{L}$, the price $P_{2}\left(q_{H}\right)$ cannot signal high quality, thus the consumer will not visit the entrant's store.

$\Rightarrow\left(p_{1}^{*}\left(q_{L}\right), p_{2}^{*}\left(q_{L}\right), p_{2}^{*}\left(q_{H}\right)\right)=\left(c_{L}, c_{L}, c_{L}+q_{H}-q_{L}\right)$.

(ii) if $\delta \leq \delta_{1}$, then

$$
\begin{aligned}
& \pi_{1}\left(c_{L}+\varepsilon, c_{L}, c_{\bar{L}}+q_{\bar{H}} \quad q_{\bar{L}}\right) \\
& 0=\pi_{1}\left(\begin{array}{llllll}
c_{L} & c_{L} & c_{L}^{+} & q_{\bar{H}} & q_{L}
\end{array}\right) \text {; } \\
& \pi_{1}\left(c_{L}-\varepsilon, c_{L}, c_{L^{+}} \quad q_{\bar{H}} \quad q_{\bar{Z}}\right. \\
& -\varepsilon<0=\pi_{1}\left(\begin{array}{llllll}
c_{L} & c_{L} & c_{L}^{+} & q_{\bar{H}} & q \text {; }
\end{array}\right. \\
& \pi_{2}\left(c_{L}, c_{L}+\varepsilon, c_{L}+q_{\bar{H}} \quad q_{\vec{L}}\right.
\end{aligned}
$$

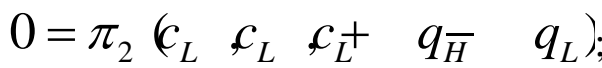

$$
\begin{aligned}
& \pi_{2}\left(c_{L}, c_{L}-\varepsilon, c_{L^{+}} \quad q_{\bar{H}} \quad q \overline{\vec{E}}\right. \\
& -\varepsilon<0=\pi_{2}\left(\begin{array}{llllll}
c_{L} & c_{L} & c_{L}^{+} & q_{\bar{H}} & q ;
\end{array}\right. \\
& \pi_{3}\left(c_{L}, c_{L}, c_{\bar{L}^{+}} \quad q_{\bar{H}} \quad \phi_{L} \varepsilon\right)= \\
& 0=\pi_{3}\left(\begin{array}{llllll}
c_{L} & c_{L} & c_{L^{+}} & q_{\bar{H}} & q_{L}
\end{array}\right) ;
\end{aligned}
$$

$$
\begin{aligned}
& \pi_{3}\left(c_{L}, c_{L}, c_{\bar{L}^{+}} \quad q_{\bar{H}} \quad q_{L} \varepsilon\right)=
\end{aligned}
$$

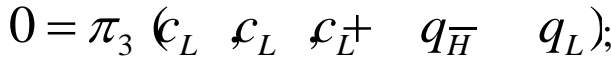

where the last two equalities are true because

$$
\begin{aligned}
& q_{L}-p_{2}^{*}\left(q_{H}\right)=q_{L}-\left(c_{L}+q_{H}-q_{L}\right)= \\
& \left(q_{L}-c_{L}\right)-\left(q_{H}-q_{L}\right)=\left(q_{L}-c_{L}\right)\left(1-\frac{q_{H}-q_{L}}{q_{L}-c_{L}}\right)= \\
& \delta_{1}\left(q_{L}-c_{L}\right) \geq \delta\left(q_{L}-c_{L}\right)=\delta\left(q_{L}-p_{1}^{*}\right)
\end{aligned}
$$

thus entry of a high-quality entrant was deterred.

$$
\Rightarrow\left(p_{1}^{*}\left(q_{L}\right), p_{2}^{*}\left(q_{L}\right), p_{2}^{*}\left(q_{H}\right)\right)=\left(c_{L}, c_{L}, c_{L}+q_{H}-q_{L}\right) \text {. }
$$

So far we have shown that for any $\delta$, there exists a separating equilibrium:

$$
\left(p_{1}^{*}\left(q_{L}\right), p_{2}^{*}\left(q_{L}\right), p_{2}^{*}\left(q_{H}\right)\right)=\left(c_{L}, c_{L}, c_{L}+q_{H}-q_{L}\right) \text {. }
$$

Next we only need to show that this equilibrium is unique (This part had been nicely done by the author).

My second concern about the paper is that, in the paper, search cost was introduced as a discount $(\delta)$ on future benefits. Intuitively we think that it should be a fixed cost, which is not related to the future benefits. The author mentioned that the way of modeling search costs was derived from Bester [2], but even in that paper Bester didn't explain why it was an appropriate way of modeling search costs. Here we will show that using a discount rate is equivalent to using a fixed amount as search cost in this case. The proof is as the following:

Suppose the search cost is given by $d$, where $d>0$. Obviously higher $d$ means higher search cost. Then Assumption 2 can be written as: Given an equilibrium price $p_{1}^{*}$, the consumer's belief satisfies $\mu\left(p_{1}^{*}, p_{2}\right)=1$ for all $p_{2}$ such that $q_{L}-p_{2}<q_{L}-p_{1}^{*}-d$, or equivalently $p_{2}>p_{1}^{*}+d$. And then Proposition 1 can be written as: Under Assumptions 1 and 2, for any $d$, there exists a unique separating equilibrium:

(i) if $d<d_{1}$, then a high-quality firm enters; $p_{1}^{*}=p_{2}^{*}\left(q_{L}\right)=c_{L}$ and $p_{2}^{*}\left(q_{H}\right)=c_{L}+q_{H}-q_{L}$; $\pi_{1}^{*}=0$ and $\pi_{2}^{*}=\alpha\left(c_{L}+q_{H}-q_{L}-c_{H}\right)$;

(ii) if $d \geq d_{1}$, then the incumbent deters entry of a high quality firm; $p_{1}^{*}=p_{2}^{*}\left(q_{L}\right)=c_{L}$ and $p_{2}^{*}\left(q_{H}\right)=c_{L}+q_{H}-q_{L} ; \pi_{1}^{*}=\pi_{2}^{*}=0$; 
where we assume $q_{H}-q_{L} \leq q_{L}-c_{L}$, and define $d_{1} \equiv q_{H}-q_{L}$.

Here we can see that with a fixed search cost, we have the exactly same equilibrium strategies and outcomes (payoffs). Therefore using a discount rate as search cost is equivalent to using a fixed amount as search cost. But the latter one makes the calculation even simpler.

Another advantage of using a fixed amount as search cost is that it makes it easier to modify the model and change it to a location game. Firstly, let us consider a simple case:

Assume we have one incumbent who produces a search good with medium quality ( $\left.q_{M}\right)$, one entrant who produce the same good with either high quality $\left(q_{H}\right)$ or low quality $\left(q_{L}\right)$. The quality of entrant's product is chosen by nature, or in other words, entrant's type is chosen by nature. The probability that entrant is a high-type is given by $\alpha$, where $\alpha \in[0,1]$. Suppose one unit of consumers uniformly distributed on a unit-length road. Incumbent locates its store on the road first (labeled as $x_{1}$ ), and then entrant chooses its location along the road to settle its store (labeled as $x_{2}$ ). Incumbent always produces medium quality product, and entrant and consumers know it. Entrant observes its own type, but incumbent and consumers do not know entrant's type. Consumer's belief of entrant's type is given by $\mu\left(x_{1}, x_{2}\left(q_{2}\right)\right)$, where $\mu \in[0,1]$. Consumers observe the locations of incumbent and entrant's stores, and decide which store to visit. If a consumer visits incumbent's store, she will buy the good there. If a consumer visits entrant's store and finds out the quality is low, she may switch to incumbent's store. But switch has a cost, which is measured by the distance between incumbent's store and entrant's store. The price of the good is fixed at $P$ and the cost of producing one good is fixed at $C$, no matter what quality the good is. Suppose that $P>C$, which means a firm makes profit if it can attract consumers to buy at its store. A consumer's utility is given by the quality of the good $(Q)$ minus the price $P$, and then minus the total route that she walked, where $Q \in\left\{q_{L}, q_{M}, q_{H}\right\}$. Finally we suppose that $q_{L}-q_{M}=q_{M}-q_{L} \equiv d_{1}, Q, P, C<<1$, and a consumer who does not buy the good from any firm will get utility $-\infty$. In this model, we can easily see that there exists a separating equilibrium such that:

(i) Incumbent locates at the center of the road $\left(x_{1}=\frac{1}{2}\right)$;

(ii) If entrant is high type, it will locate at the same position as where incumbent locates $\left(x_{2}=x_{1}\right)$; if entrant is low type, it will locate at $x_{2}=x_{1}+d_{1}$ if $x_{1} \leq \frac{1}{2}$, or $x_{2}=x_{1}-d_{1}$ if $x_{1}>\frac{1}{2}$ (equivalent to $x_{2}=x_{1}+d_{1} *(-1)^{I\left(x_{1}>\frac{1}{2}\right)}$ );

(iii) Consumers' beliefs $\mu\left(x_{1}, x_{2}\left(q_{2}\right)\right)=1$ if $\left|x_{1}-x_{2}\right|<d_{1}$.

Proof: Let $\varepsilon$ be arbitrarily small and $0<\varepsilon<d_{1}$, we have

$$
\begin{aligned}
& \pi_{1}\left(\frac{1}{2}-\varepsilon ; \frac{1}{2}-\varepsilon+d_{1}, \frac{1}{2}-\varepsilon\right)=(1-\alpha)\left(\frac{1}{2}-\varepsilon+d_{1}\right)(P-C) \\
& <(1-\alpha)\left(\frac{1}{2}+d_{1}\right)(P-C)=\pi_{1}\left(\frac{1}{2} ; \frac{1}{2}+d_{1}, \frac{1}{2}\right) ;
\end{aligned}
$$$$
\pi_{1}\left(\frac{1}{2}+\varepsilon ; \frac{1}{2}+\varepsilon-d_{1}, \frac{1}{2}+\varepsilon\right)=(1-\alpha)\left(\frac{1}{2}-\varepsilon+d_{1}\right)(P-C)
$$$$
<(1-\alpha)\left(\frac{1}{2}+d_{1}\right)(P-C)=\pi_{1}\left(\frac{1}{2} ; \frac{1}{2}+d_{1}, \frac{1}{2}\right) ;
$$$$
\pi_{2}\left(\frac{1}{2} ; \frac{1}{2}+d_{1}-\varepsilon, \frac{1}{2}\right)=0<\left(\frac{1}{2}-d_{1}\right)(P-C)
$$$$
=\pi_{2}\left(\frac{1}{2} ; \frac{1}{2}+d_{1}, \frac{1}{2}\right) \text {; }
$$$$
\pi_{2}\left(\frac{1}{2} ; \frac{1}{2}+d_{1}+\varepsilon, \frac{1}{2}\right)=\left(\frac{1}{2}-d_{1}-\varepsilon\right)(P-C)
$$$$
<\left(\frac{1}{2}-d_{1}\right)(P-C)=\pi_{2}\left(\frac{1}{2} ; \frac{1}{2}+d_{1}, \frac{1}{2}\right) \text {; }
$$

$\pi_{3}\left(\frac{1}{2} ; \frac{1}{2}+d_{1}, \frac{1}{2}-\varepsilon\right)=(P-C)=\pi_{3}\left(\frac{1}{2} ; \frac{1}{2}+d_{1}, \frac{1}{2}\right) ;$

$\pi_{3}\left(\frac{1}{2} ; \frac{1}{2}+d_{1}, \frac{1}{2}+\varepsilon\right)=(P-C)=\pi_{3}\left(\frac{1}{2} ; \frac{1}{2}+d_{1}, \frac{1}{2}\right)$.

$\Rightarrow$ None of the players has incentive to deviate form the equilibrium strategies

$$
\left(x_{1}=\frac{1}{2} ; x_{2}\left(q_{L}\right)=x_{1}+d_{1} *(-1)^{I\left(x_{1}>\frac{1}{2}\right)}, x_{2}\left(q_{H}\right)=x_{1}\right) \text {. }
$$

In the above equilibrium, incumbent and entrant's expected payoffs are 


$$
\begin{gathered}
\pi_{1}^{*}=(1-\alpha)\left(\frac{1}{2}+d_{1}\right)(P-C), \\
\pi_{2}^{*}=\alpha(P-C)+(1-\alpha)\left(\frac{1}{2}-d_{1}\right)(P-C),
\end{gathered}
$$

respectively. Also, we can see that in this setup, high-quality entrant can signal its quality by locating its store very close to incumbent's store (distance smaller than $d_{1}$ ).

However, if we assume that incumbent observes entrant's type (similar to the original Model II), then we will get the same separating equilibrium - it won't alter any player's strategies. Thus we may consider a more interesting model which will give us different separating equilibrium strategies under different assumptions about incumbent's awareness of entrant's type. The new location game is similar to the previous one except now incumbent has two stores along the road, which means incumbent locates two stores $\left(x_{1}^{1} \& x_{1}^{2}\right)$ in the market first and then entrant chooses a location $\left(x_{2}\right)$ to settle its store. We assume incumbent will symmetrically locate its two stores (i.e., $x_{1}^{2}=1-x_{1}^{1}$ ). Under the assumption that incumbent does not observe entrant's type, there exists a separating equilibrium such that:

(i) Incumbent locates two stores at $x_{1}^{1}=\frac{1}{4}$ and $x_{1}^{2}=\frac{3}{4}$;

(ii) For a low-type entrant, $x_{2}=\frac{1}{2}$ if $x_{1}^{1} \leq \frac{1}{4}$, and $x_{2}=x_{1}^{1}-d_{1}$ if $x_{1}^{1}>\frac{1}{4}$; for a high-type entrant, $x_{2}=x_{1}^{1}+d_{1}$ or $x_{2}=x_{1}^{2}-d_{1}$;

(iii) Consumers' beliefs $\mu\left(x_{1}^{1}, x_{1}^{2}, x_{2}\left(q_{2}\right)\right)=1$ if $x_{2}-x_{1}^{1}=d_{1}$ or $x_{1}^{2}-x_{2}=d_{1}$.

Proof: Let $\varepsilon$ be arbitrarily small and $0<\varepsilon<d_{1}$, we have $\pi_{1}\left(\left\{x_{1}^{1}=\frac{1}{4}-\varepsilon, x_{1}^{2}=\frac{3}{4}+\varepsilon\right\} ; x_{2}\left(q_{L}\right)=\frac{1}{2}, x_{2}\left(q_{H}\right)=\frac{1}{4}-\varepsilon+d_{1}\right)=$ $\alpha\left(\frac{1}{2}-d_{1}\right)+(1-\alpha)\left(\frac{3}{4}+d_{1}-\varepsilon\right)<\alpha\left(\frac{1}{2}-d_{1}\right)+(1-\alpha)\left(\frac{3}{4}+d_{1}\right)=$ $\pi_{1}\left(\left\{x_{1}^{1}=\frac{1}{4}, x_{1}^{2}=\frac{3}{4}\right\} ; x_{2}\left(q_{L}\right)=\frac{1}{2}, x_{2}\left(q_{H}\right)=\frac{1}{4}+d_{1}\right) ;$ $\pi_{1}\left(\left\{x_{1}^{1}=\frac{1}{4}+\varepsilon, x_{1}^{2}=\frac{3}{4}-\varepsilon\right\} ; x_{2}\left(q_{L}\right)=\frac{1}{4}+\varepsilon-d_{1}, x_{2}\left(q_{H}\right)=\frac{1}{4}+\varepsilon+d_{1}\right)=$ $\alpha\left(\frac{1}{2}-d_{1}\right)+(1-\alpha)\left(\frac{3}{4}+d_{1}-\varepsilon\right)<\alpha\left(\frac{1}{2}-d_{1}\right)+(1-\alpha)\left(\frac{3}{4}+d_{1}\right)=$ $\pi_{1}\left(\left\{x_{1}^{1}=\frac{1}{4}, x_{1}^{2}=\frac{3}{4}\right\} ; x_{2}\left(q_{L}\right)=\frac{1}{2}, x_{2}\left(q_{H}\right)=\frac{1}{4}+d_{1}\right) ;$ $\pi_{2}\left(\left\{\frac{1}{4}, \frac{3}{4}\right\} ; \frac{1}{2}-\varepsilon, \frac{1}{4}+d_{1}\right)=\frac{1}{4}-d_{1}=\pi_{2}\left(\left\{\frac{1}{4}, \frac{3}{4}\right\} ; \frac{1}{2}, \frac{1}{4}+d_{1}\right) ;$ $\pi_{2}\left(\left\{\frac{1}{4}, \frac{3}{4}\right\} ; \frac{1}{2}+\varepsilon, \frac{1}{4}+d_{1}\right)=\frac{1}{4}-d_{1}=\pi_{2}\left(\left\{\frac{1}{4}, \frac{3}{4}\right\} ; \frac{1}{2}, \frac{1}{4}+d_{1}\right) ;$ $\pi_{3}\left(\left\{\frac{1}{4}, \frac{3}{4}\right\} ; \frac{1}{2}, \frac{1}{4}+d_{1}-\varepsilon\right) \leq \frac{1}{2}+d_{1}=\pi_{3}\left(\left\{\frac{1}{4}, \frac{3}{4}\right\} ; \frac{1}{2}, \frac{1}{4}+d_{1}\right) ;$ $\pi_{3}\left(\left\{\frac{1}{4}, \frac{3}{4}\right\} ; \frac{1}{2}, \frac{1}{4}+d_{1}+\varepsilon\right)=\frac{1}{2}+d_{1}=\pi_{3}\left(\left\{\frac{1}{4}, \frac{3}{4}\right\} ; \frac{1}{2}, \frac{1}{4}+d_{1}\right)$. $\Rightarrow$ None of the players has incentive to deviate from the equilibrium strategies

$$
\left(\left\{x_{1}^{1}=\frac{1}{4}, x_{1}^{2}=\frac{3}{4}\right\} ; x_{2}\left(q_{L}\right)=\frac{1}{2}, x_{2}\left(q_{H}\right)=\frac{1}{4}+d_{1}\right) .
$$

In the above equilibrium, incumbent and entrant's expected payoffs are

$$
\begin{aligned}
& \pi_{1}^{*}=\left[\alpha\left(\frac{1}{2}-d_{1}\right)+(1-\alpha)\left(\frac{3}{4}+d_{1}\right)\right](P-C) \\
& \pi_{2}^{*}=\left[\alpha\left(\frac{1}{2}+d_{1}\right)+(1-\alpha)\left(\frac{1}{4}-d_{1}\right)\right](P-C)
\end{aligned}
$$

and

respectively. Also, we can see that in this setup, high-quality entrant can signal its quality by locating its store between incumbent's two stores and close to one of them (with distance $d_{1}$ ).

Under the assumption that incumbent observes entrant's type, and consumers know incumbent observes entrant's type, there exists a separating equilibrium such that:

(i) If entrant is a low-type, incumbent locates two stores at $x_{1}^{1}=\frac{1}{4}$ and $x_{1}^{2}=\frac{3}{4}$; entrant locates at

$$
\begin{aligned}
& x_{2}=\frac{1}{2} \quad \text { if } \quad x_{1}^{1} \leq \frac{1}{4}, \text { and } x_{2}=x_{1}^{1}-d_{1} \text { if } \\
& x_{1}^{1}>\frac{1}{4}
\end{aligned}
$$


(ii) If entrant is a high-type, incumbent locates two stores at $x_{1}^{1}=0$ and $x_{1}^{2}=1$; entrant locates at $x_{2} \in\left[x_{1}^{1}+d_{1}, x_{1}^{2}-d_{1}\right]$;

(iii) Consumers' beliefs $\mu\left(x_{1}^{1}, x_{1}^{2}, x_{2}\left(q_{2}\right)\right)=1$ if $x_{2}-x_{1}^{1}=d_{1}$, or $x_{1}^{2}-x_{2}=d_{1}$, or $x_{1}^{1}=0$.

The proof of the above separating equilibrium is similar to the previous cases. Here we can see that since incumbent's locations of stores can signal entrant's type, a high-quality entrant has more freedom to choose its location.

\section{SUMMARY}

Another possible extension of de Bijl's paper would be to consider the following scenario: Entrant is high-quality type and it signals its type by $p_{2}\left(q_{H}\right)$. Incumbent observes the entrant's type but it signals entrant as a low-quality type by $p_{1}\left(q_{L}\right)$. Then consumers cannot distinguish if it is a low-quality entrant mimicking a high type or it is the incumbent intentionally deviating from equilibrium price. In other words, consumers do not know who is lying. In this case the separating equilibrium will depend on the search cost $(\delta)$. The author evaded this situation in the paper by assuming the price vector $\left(p_{1}, p_{2}\right)$ was either weakly consistent with $q_{L}$ but not with $q_{H}$, or weakly consistent with $q_{H}$ but not with $q_{L}$. It may be an interesting case and worth further pursuit.

\section{REFERENCES}

[1] De Bijl, Paul W. J. (1997). Entry deterrence and signaling in markets for search goods. International Journal of Industrial Organization, Vol. 16, Page 1-19.

[2] Bester, Helmut (1993). Bargaining versus price competition in markets with quality uncertainty. American Economic Review, Vol. 83, No. 1, Page 278-288. 